\title{
HISTO-MORPHOMETRIC STUDY OF VARIOUS SEGMENTS OF VERTEBRAL ARTERY IN HUMAN CADAVERS
}

\author{
Ashwini R Desai *, S.K. Chavan.
}

Rural MedicalCollege PMT, Loni, Dist: Ahmednagar, Maharashtra, India

\section{ABSTRACT}

Background: Vertebral artery is a medium sized artery which has long, tortuous course through neck and cranium. It is highly protected by bony and muscular structures because it provides the vascularization to important intracranial structures. During cervical spine movements, it is exposed to large shear and tensile forces at the exit from second cervical vertebra. A reduction in blood flow in vertebral artery may occur due to many congenital and structural factors which will cause ischemia and various neurological symptoms. Vertebral artery tears after blows to the head and neck are usually associated with basal subarachnoid haemorrhage with a rapidly fatal outcome. Aim of study was to study histomorphometric variations in four segments of vertebral artery.

Material and methods: All four segments of vertebral arteries each having $0.5 \mathrm{~cm}$ long of both sides were collected from 30 cadavers. Outer and inner diameters were measured. Tissues were processed and stained by Verhoeff's Van Geison and Masson Trichrome. Slides were scanned by "IS capture software" under $10 \mathrm{X}$ magnifications.

Results: Outer diameter of left vertebral artery was $3.598 \pm 0.32 \mathrm{~mm}$ and that of right was $3.286 \pm 0.33 \mathrm{~mm}$. Inner diameter of left vertebral artery was $3.19 \pm 0.30 \mathrm{~mm}$ and right was $2.86 \pm 0.26 \mathrm{~mm}$. Diameter of $1^{\text {st }}$ and $3^{\text {rd }}$ part of vertebral artery was more than $2^{\text {nd }}$ and $4^{\text {th }}$ part. Internal elastic lamina was thick and prominent in $4^{\text {th }}$ part. External elastic membrane was prominent in $1^{\text {st }}, 2^{\text {nd }}$, and $3^{\text {rd }}$ part, but it is completely absent or represented by sparse fibrils in $4^{\text {th }}$ part of vertebral artery.

Conclusion: Left vertebral artery was found to be dominant than the right.Internal elastic lamina was main elastic constituent of $4^{\text {th }}$ part of vertebral artery, so damage to it may cause vascular pathologies like atherosclerosis, aneurysm etc.

KEY WORDS: Vertebral artery, Inner diameter, Outer diameter, Internal elastic membrane, External elastic membrane.

Address for Correspondence: Dr. Ashwini R Desai, Department of Anatomy, Rural Medical College, PIMS, DU. Loni, Dist: Ahmednagar, Maharashtra, India, Pin: 413736

Mobile no-+919975493999 E-Mail: drrajeevdesai31@yahoo.in

\section{Quick Response code}

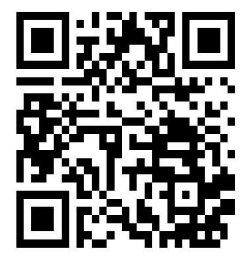

DOI: $10.16965 /$ ijar.2018.415
Journal Information

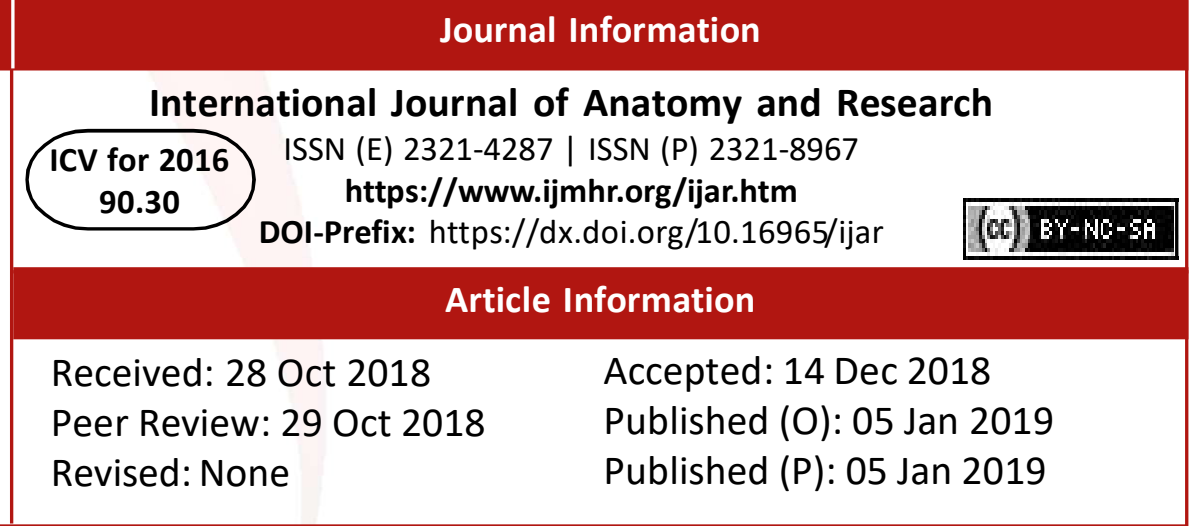

\section{INTRODUCTION}

Vertebral artery (VA) arises from the posterolateral aspect of the first part of the subclavian artery. It is the medium sized artery which has long, tortuous course through neck and cranium [1]. Vertebral arteries are important arteries as they supply the hind brain centers that control the respiratory, cardiac and equilibrium functions $[1,2]$. VA has four parts:

V1: The first part passes backwards and upwards between the longus coli and scalenus anterior muscles up to transverse foramen of $6^{\text {th }}$ cervical vertebra.

V2: The second part ascends through the 
transverse foramen of upper six cervical vertebrae. Here it is accompanied by branch of inferior cervical ganglion and plexus of veins.

V3: The third part passes backwards and medially behind the lateral mass of atlas. This part lies in sub-occipital triangle.

V4: The fourth part pierces the dura and arachnoid mater. It ascends anterior to hypoglossal roots. It inclines anterior to the medulla oblongata to meet with VA of opposite side to form the basilar artery. [1, 3].

The vertebral artery is highly protected by bony and muscular structures because it provides the vascularization to important intracranial structures.During cervical spine movements,VA is exposed to large shear and tensile forces at the exit from second cervical vertebra. It is elongated during lateral flexion and kinking occurs during rotation of neck $[4,5]$. However, vertebral arteries are continuously exposed to stress and strain still the damage to the artery is minimal. The mechanism of protection of these arteries may lie in the structure of its wall [6].

A reduction in blood flow in the VA may occur due to many congenital and structural factors which will cause ischemia and various neurological symptoms.

VA may be compressed from outside due to exostoses of the cervical vertebrae and athero scleroticplaques in the wall of the artery can be aninternal cause of compromised blood flow in the artery.Also, full range rotation of cervical spine may cause mechanical stretching of the VA and it willdecrease the blood flow through it. Initial size of the artery play important role in decreased blood flow $[3,7,8]$.

VA tears after blows to the head and neck are usually associated with basal subarachnoid hemorrhage with a rapidly fatal outcome [6].

Precise knowledge of anatomy and variations of VA is mandatory while performing angiography and various surgical procedures to prevent serious complications[9]. While searching the literature, we observed that only few workers have studied the histo-morphometry of various segments of vertebral artery in human cadavers. Considering the clinical importance and lack of paucity in the literature we worked on present topic.

\section{MATERIALS AND METHODS}

Present study was done on thirty human cadavers of unknown age and sex which were available in department of anatomy. These cadavers were used by undergraduate students for routine dissection. Permission was obtained from the Institutional Ethical committee. Meticulous dissection of the VA throughout its length was done on both sides. All four segments of VA, each having $0.5 \mathrm{~cm}$ lengths were taken. Outer and inner diameters were measured by Verniercalliper in $\mathrm{mm}$ and kept in separate containers in $10 \%$ formalin. Each of the containers was properly labeled and tissue stored till it was processed.

Four different sites of VA from where the sample was taken:

- $1^{\text {st }}$ part (V1) $-1 \mathrm{~cm}$ above its origin from subclavian artery.

- $2^{\text {nd }}$ part (V2) -in between the transverse process of $\mathrm{C}_{3}$ to $\mathrm{C}_{5}$ vertebra.

- $3^{\text {rd }}$ part (V3) -in sub-occipital triangle.

- $4^{\text {th }}$ part (V4) $-1 \mathrm{~cm}$ proximal to formation of basilar artery.

Tissues were processed through routine procedure and blocks prepared. Five to seven micro meters thick sections were cut and were stained with Verhoeff's Van Geison, and Masson Trichrome[10]. Stained slides were scanned by IS capture software under $10 \mathrm{X}$ magnification.

Normal structure and variations in histology of the various parts of VA were observed, recorded and where needed photomicrography was done.

Following histological parameters were measured:

a. Whole thickness of arterial wall inmicrometre

b. Thickness of tunica media (TM) in micrometre

c. Appearance of internal and external elastic lamina (IEL and EEL)

d. Disposition of elastic and muscle fibres in TM e. Disposition of elastic and collagen fibres in tunica adventitia (TA)

\section{RESULTS}

Present study observed that outer diameter of vertebral artery on left side is $3.598 \mathrm{~mm} \pm 0.32$ 
and on right side is $3.286 \mathrm{~mm} \pm 0.33$. unpaired $t$-test ( $t=3.659)$ shows that mean difference in outer diameter of left and right side of vertebral artery is $0.3018,95 \% \mathrm{Cl}$ lower limit of which is 0.1408 andupper limit is 0.4809 and $p$ value is 0.0005 which is less than 0.05 and hence difference is significant. (Table 1).

Present study observed that inner diameter of vertebral artery on left side is $3.19 \mathrm{~mm} \pm 0.30$ and on right side is $2.86 \mathrm{~mm} \pm 0.26$. Unpaired $t$-test ( $t=4.507)$ shows thatmean difference in inner diameter of left and right side of vertebral artery is $0.3303,95 \% \mathrm{Cl}$ lower limit of which is 0.1836 andupper limit is 0.4771 and $p$ value is 0.0001 which is less than 0.05 and hence difference is significant. [Table 1]

Table 1: Segment wise mean diameter of vertebral artery (mm).

\begin{tabular}{|c|c|c|c|c|c|c|c|c|c|c|}
\hline Diameter & \multicolumn{2}{|c|}{ V1 } & \multicolumn{2}{c|}{ V2 } & \multicolumn{2}{c|}{ V3 } & \multicolumn{2}{c|}{ V4 } & \multicolumn{2}{c|}{ Mean of all 4 } \\
\hline & Right & Left & Right & Left & Right & Left & Right & Left & Right & Left \\
\hline Outer & 3.862 & 4.107 & 3.308 & 3.671 & 3.375 & 3.632 & 2.602 & 2.979 & 3.286 & 3.598 \\
\hline Inner & 3.265 & 3.644 & 2.915 & 3.313 & 2.929 & 3.183 & 2.329 & 2.62 & 2.859 & 3.19 \\
\hline
\end{tabular}

Present study observed the variability in the thickness of the VAs and V4 has the thinnest wall (Table 2).

Table 2: Segment wise thickness of arterial wall of vertebral artery in micrometer.

\begin{tabular}{|c|c|c|c|c|c|c|c|c|c|}
\hline \multicolumn{2}{|c|}{ V1 } & \multicolumn{2}{c|}{ V2 } & \multicolumn{2}{c|}{ V3 } & \multicolumn{2}{c|}{ V4 } & \multicolumn{2}{c|}{$\begin{array}{c}\text { Mean of all 4 } \\
\text { segments }\end{array}$} \\
\hline Right & Left & Right & Left & Right & Left & Right & Left & Right & Left \\
\hline 426.09 & 430.12 & 343 & 352.06 & 423.52 & 424.43 & 220.26 & 251.43 & 353.21 & 364.51 \\
\hline
\end{tabular}

In V1 part of VA: we observed that the endothelium was seen to be discontinuous in some specimens; subendothelial tissue (SET) show variable thickness.Internal elastic lamina (IEL) was prominent and wavy.Tunica media (TM) was thick and comprise of smooth muscle, collagen and elastic fibre. The distribution of elastic fibre was found to be more towards inner segment of TM than outer segment (average of 2-3 elastic lamellae). Rest part is ofmuscle andcollagen fibres. External elastic lamina (EEL) shows single lamina of circularly arranged elastic fibres and it was surrounded by many layers of longitudinally arranged elastic fibres. In tunica adventitia (TA) concentration of elastic fibres are more as compared to collagen and mostly longitudinally arranged with few circularly arranged. [Fig.1].
Fig. 1: Photomicrograph of first part of vertebral artery showing Tunica Intima (TI), Internal Elastic Lamina,Tunica Media (TM), External Elastic Lamina (EEL),Tunica Adventitia (TA). Blue arrows showing elastic fibres in TM which are close to IEL(under 10X magnification)

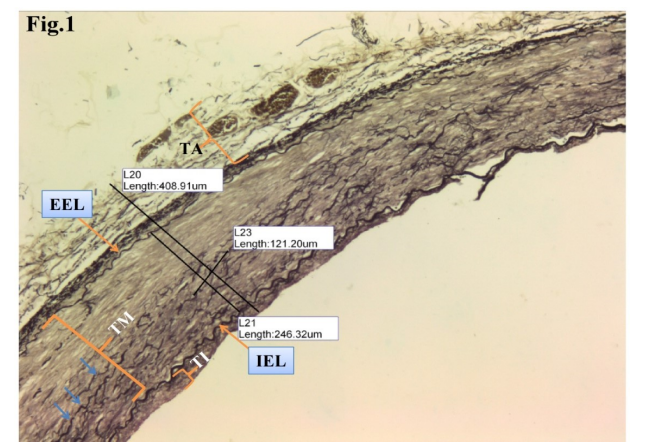

In V2 part of VA: the endothelium and SET were well appreciated with variable thickness. Thin TI was observed compared V1 and V3. IEL was prominent. TM was less thick as compared to $1^{\text {st }}$ part of VA and was muscular. Numbers of elastic fibres were less as compared to V1.EEL shows 3 to 5 layers of circularly arranged elastic fibres. TA shows circularly arranged bundles of collagen fibres prominently with very few elastic fibres[Fig. 2A, and B].

Fig. 2A: Photomicrograph of second part of vertebral artery showing Tunica Intima ( $\mathrm{TI})$, Internal Elastic Lamina,Tunica Media (TM), External Elastic Lamina (EEL),Tunica Adventitia (TA) [MassonsTrichrome stain](under 10X magnification)

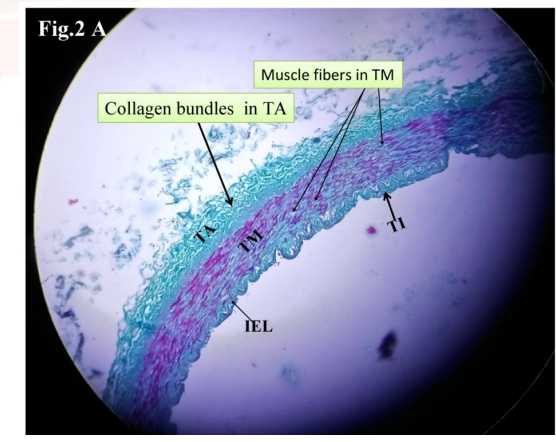

Fig. 2B: Photomicrograph of second part of vertebral artery showing Tunica Intima (TI), Internal Elastic Lamina,Tunica Media (TM), External Elastic Lamina (EEL),Tunica Adventitia (TA) [Verhoeff's van- Geisons stain]. (under $10 \mathrm{X}$ magnification)

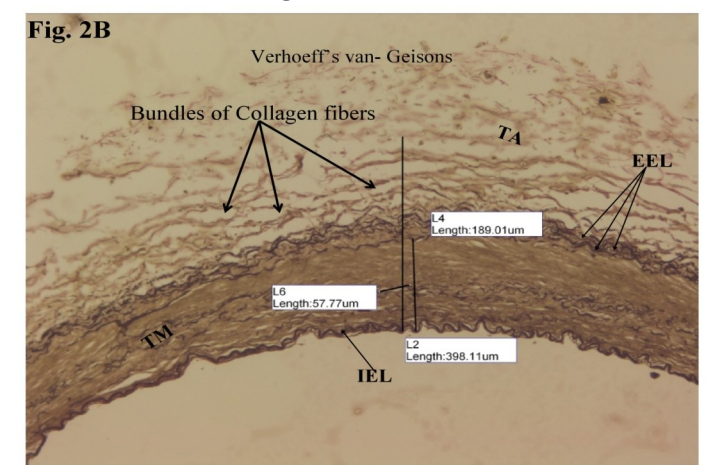


In V3 part of VA: the endothelium and SET were distorted at places and well appreciated with variable thickness at some places. IEL was prominent and wavy. TM was thick than rest of the parts of VA and mainly consist of smooth muscle interspersed in between are elastic fibrils. At some places it also showed fragmented elastic fibres.EEL is prominent with inner circular and outer longitudinally arranged elastic fibres.TA shows more elastic fibres as compared to V2 and V4 segments.

Fig. 3A and B: Photomicrograph of third part of vertebral artery showing Tunica Intima (TI), Internal Elastic Lamina,Tunica Media (TM), External Elastic Lamina (EEL),Tunica Adventitia (TA) [Verhoeff's van- Geisons stain].(under 10X magnification)

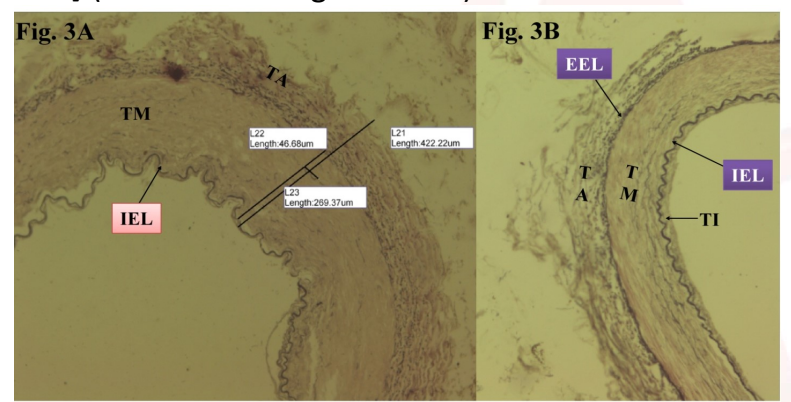

In V4 part of VA: IEL was very prominent, thick and less wavy as compared to other three parts of VA. TM is thin as compared to other segments. It contains comparatively more number of muscle fibres than elastic fibres. EEL was either absent or it was represented by sparse single elastic fibrils only at some places. TA was thin and shows mainly collagen fibres. We observed thick tunica media in third part of vertebral artery (Table no- 3 )

Table 3: Segment wise thickness of tunica media of vertebral artery in micrometre.

\begin{tabular}{|c|c|c|c|c|c|c|c|c|c|}
\hline \multicolumn{2}{|c|}{ V1 } & \multicolumn{2}{|c|}{ V2 } & \multicolumn{2}{c|}{ V3 } & \multicolumn{2}{c|}{ V4 } & \multicolumn{2}{c|}{$\begin{array}{c}\text { Mean of all 4 } \\
\text { segments }\end{array}$} \\
\hline Right & Left & Right & Left & Right & Left & Right & Left & Right & Left \\
\hline 209.78 & 211.201 & 157.95 & 160.421 & 226.59 & 230.34 & 126.82 & 128.53 & 180.28 & 182.62 \\
\hline
\end{tabular}

Fig. 4A: Photomicrograph of fourth part of vertebral artery showing Internal Elastic Lamina,Tunica Media (TM), External Elastic Lamina (EEL) represented by sparse single elastic fibrils, Tunica Adventitia (TA) [Verhoeff's van- Geisons stain](under 10X magnification)

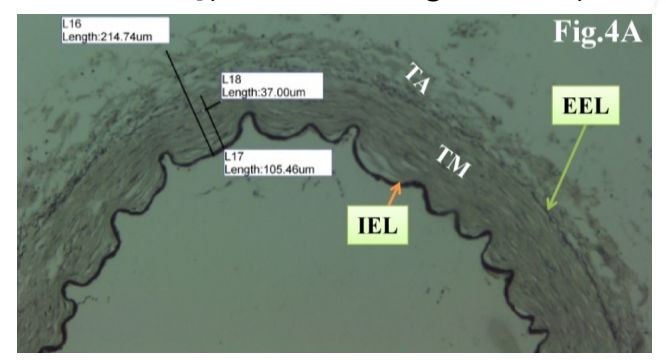

Int J Anat Res 2019, 7(1.1):6102-07. ISSN 2321-4287
Fig. 4 B: Photomicrograph of fourth part of vertebral artery showing Tunica Intima (TI),Tunica Media (TM), External Elastic Lamina (EEL)- absent, Tunica Adventitia (TA) [Verhoeff's van- Geisons stain](under 10X magnification).

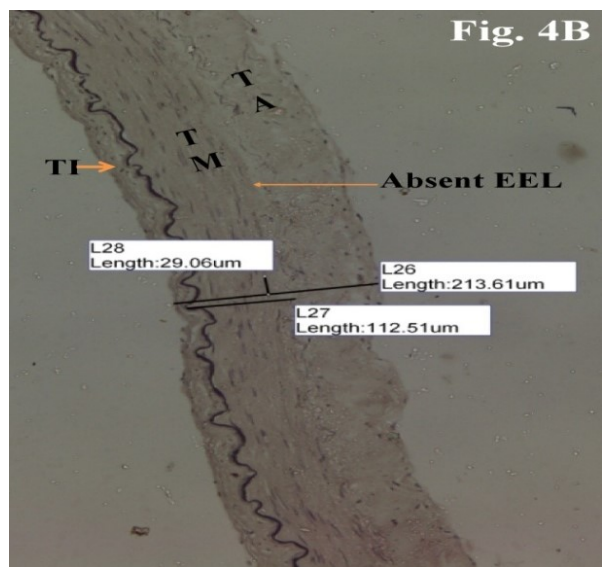

\section{DISCUSSION}

VA has a very unique, long tortuous course through the neck and cranium [1]. VAs is continuously exposed to stress and strain. At the point of exit from C2 vertebra they are exposed to large shear and tensile forces during cervical spine movements. In spite of all these adverse conditions, damage to artery is very less and the mechanism for protection lies in the structure of the arterial wall [4]. According to most of anatomical text books and researchers the left VA is larger than right [1, 2, 4, 7,]. Present study also observed outer and inner mean diameter of left VA was higher than right. Diameter of the VA was seen more in V1 and V3 segment than V2 and V4 segment which is (Our findings are comparable with the findings) contradictory to the findings of Bhadkaria et al. [11]. Bloom and Fawcett mentioned that intracranial arteries are thin walled as they are protected by external pressure and tension within the cranial cavity [12].

In addition, Leesson TS et al. states that intracranial arteries have well developed internal elastic membrane[13]. In present study also $4^{\text {th }}$ part of vertebral artery is thin walled with prominent IEL. Striking histological changes occurred in IV part of VA that it becomes thin with decreased elastic fibers in TA and Tunica media. EEL is absent or found in the form of sparse fibrils.

Tunica intima: In the present study, the endothelial lining of the artery was seen to be discontinuous at some places. This may be due 
to damage during processing. Sub endothe-lial tissue showed variable thickness in different segments of the artery on both the sides (Fig. $1,2,3,4)$.Sato et al. observed thick tunica intima in $4^{\text {th }}$ part of VA [14] but Wilkinson did not found difference in TI of extracranial and intracranial segment of vertebral artery[15]. Present study observed thin $\mathrm{TI}$ in $2^{\text {nd }}$ part of VA (Fig. $2 \mathrm{~A}$ and B).Muscular arteries have thin $\mathrm{TI}$, endothelium being directly resting on internal elastic lamina[16 ]. Present study shows thick sub endothelial tissue observed in Ist and III part VA may be due to age related adaptive changes [17].

Internal Elastic Lamina: It is in accordance with the features of muscular. prominent and wavy.We observed variability in thickness and disposition of IEL. It was very wavy at places and at other it is less wavy particularly in IV part. Bhadkaria et al observed double layered IEL in few cases [11].IEL is thick and is the main elastic constituent of $4^{\text {th }}$ part of vertebral artery and may be sufficient elastic component to maintain transmitted blood pressure. But Wilkinson described it was more obvious in the intradural segment as the rest of the arterial wall is thinner and there was very little elastic tissue in the arterial wall [15].

Tunica media: TM wall thickness is related to the flow dynamics. S. Cavdar et al. stated that thickness of tunica media is reduced in V2 segment above $\mathrm{C} 5$ foramen and then gradually increased and highest at $C 1$. [18]. In present study TM of V3 segment was also thickest of all other segment.

Johnson et al. found elastic count in TM gradually decreases along the course of artery. Wilkinson mentioned that medial thickness of intradural segment is noticeably less than extradural part [14]. Our findings are in correlation with these findings. In the present study tunica media of Ist part of VA showed more of elastic fiber in inner segment than its outer segment. This finding may be the just the gradual change with reduction of elastic fibre from outer segment of TM in a process towards themuscular artery as it moves away from heart [19]. Chopard et.al also noted similarly that elastic fiber is found to be more in the internal portion of tunica media [20].
TM of V3 segment of vertebral artery comparatively dominated by smooth muscle and relatively less number of elastic fibre when compared with I and II part of VA. TM of V4 segment had abundant of smooth muscle fibres. This pattern was contradictory to finding of Bhadkaria et al.[11].

External Elastic Lamina and Tunica Adventitia: According to Wilkinson I, II, III part of vertebral arteryshowed well developed adventitia and an underlying thick elastic lamina. VAs had unchanged structure from its origin to a point 1 $\mathrm{cm}$ proximal to dural perforation. After penetrating dura, VA has less thick TA and EEL is either absent or show sparsefibrils. Coast and Gee and Winckler described inner circular and outer longitudinal arrangement of elastic fiber in tunica adventitia[21,22].

Present study observed well defined TA in all segments of VA with variable thickness. V1 and V3 part showsmore elastic fibre in tunica adventitia this may allow the stretching of VA during rotatory movements.

Present study may help in understanding the relation between the amount of blood flow and wall thickness of VA. This study is helpful to understand the changes occurring in different parts of artery and pathology occurring in particular part.

\section{CONCLUSION}

The outer and inner diameters of left sided vertebral artery are more than right side and is statistically significant.Diameter of vertebral artery is more in V1 and V3 than V2 and V4 segment. TI thin in V2 segment. TM was thick in V3 segment. Thick Tunica media in III part of vertebral artery may regulate blood flow during the head rotation. IEL was observed in all segments of VA and was thick and prominent in V4 segment. IEL is the main elastic constituent of V4 segment of vertebral artery so damage to it may cause vascular pathologies like aneurysms. EEL in V4 segment is either absent or represented by sparse fibrils. More detail study should be done on different age groups, known sex and different ethnic groups. Also different types of stains can be used to observe the details of fibres. 


\section{ABBREVIATIONS}

VA- vertebral artery

TI- Tunica intima

TM- Tunica media,

TA- Tunica adventitia

IEL- internal elastic lamina

EEL- external elastic lamina

SET- subendothelial tissue.

\section{Conflicts of Interests: None}

\section{REFERENCES}

[1]. Standrings S, Neil RB, Patricia C, et al. Eds; Chapter28 neck In: Gray's Anatomy, 40th Ed, Churchill Livingstone Elsevier. Edinburg. 2008; 449.

[2]. Rawal JD, Jadav HR. Histomorphometric comparison of diameter of right and left vertebral arteries. National Journal of Medical Research. 2012; 02(03):260-263.

[3]. Romanes G J. Cunningham's Manual of Practical Anatomy. $15^{\text {th }}$ edition. New York: Oxford Medical Publications, 2000; 216.

[4]. Kornieieva MA, Al-Hadidi AM. Morphology of the vertebral artery in Asian Population. Asian Journal of Medical Sciences. 2014;5(4):084-088,

[5]. Park KW, Park JS, Hwang SC, et al. Vertebral artery dissection: natural history, clinical features and therapeutic considerations. Journal of Korean Neurosurgical Society. 2008;44(3):109-115.

[6]. Johnson CP, Baugh R, Wilson CA, et al. Age related changes in the tunica media of the VA: implications for the assessment of vessels injured by trauma. Journal of clinical pathology. 2001;54:139-145.

[7]. Mitchell J, McKay A. Comparison of left and right vertebral artery intra-cranial diameters. Anatomical Record 1995; 242: 1350-1354.

[8]. Macchi C, Giannelli F, Cecchi F, Gulisano M, Pacini $P$, Corcos L, Catini C, Brizzi E. The inner diameter of human intra-cranialvertebral artery by color Doppler method. Italian Journal of Anatomy and Embryology 1996; 10l: 81-87

[9]. Sikka A, Jain A. Bilateral variation in the origin and course of the vertebral artery. Hindawi Publishing Corporation Anatomy Research International. 2012;12:01-03.
[10]. Bancroft JD and Gamble M. Eds; Chapter-The hematoxylins and eosin, connective tissues and stains: In theory and practice of histological techniques, 6th Ed, Churchill Livingstone Elsevier. 2008;122123, 146-153.

[11].BhadkariaV, Chawre H K, S. S. Joshi SS, JoshiSD. Histological variations in various segments of vertebral artery. J E M D. 2016; 5 (2): 120-26.

[12]. Bloom and Fawcett.chapter-12, Blood and lymph vascular system. In: A textbook of histology,12 th Ed, chapman and Hall, New York, London.1994; 377.

[13]. Leeson TS, Leeson CR, Paparo AA, chapter- 8, The circulatory system. In: Text /Atlas of histology, W. B. Saunders international edition. 1988; 319.

[14]. Sato T, Sasaki T, Suzuki K, et al. Histological study of the normal vertebral artery etiology of dissecting aneurysms. Neurol Med Chir (Tokyo). 2004; 44:629636.

[15]. Wilkinson IMS The vertebral artery; intracranial and extracranial structure. Arch Neurol 1972; 27:392-396.

[16]. Ross MH, Reith EJ, chapter- 12,Cardiovascular system, In: Histology-A text and atlas, Harper international edition, 1985; 283-284.

[17]. Khan MM, Butt SB, Bashir Kiani MR. Age related changes in histomorphology of medium sized muscular artery. Pak Armed Forces Med J 2014;64 (4): 609-13

[18]. CavdarS, Dalchik H, Ercan F, Arbak S, Arifoglu Y. A morphological study of the V2 segment of vertebral artery. Okajimas Folia Anat. Jpn. August 1996; 73(23):133-138.

[19]. Kumar Keshaw. Microstructure of human arteries. Journal of Anatomical society of India 2001;3:137140.

[20]. Chopard R P, Lucas G, Laudana A. Microscopic anatomy of the human vertebro-bacilar system. Arq Neuro-Psiquiat (San Paulo) 1991;49(4);430-3.

[21].Coast GC, Gee DJ. Traumatic subarachnoid haemorrhage-an alternative source.J Clin Pathol 1984;37: 1245-8.

[22].Winckler G. Remarques sur la structure de l'arterevertebrale.Quaderni di AnatomiaPractica S XXV111 1972; N1-4:105-15.

How to cite this article:
Ashwini R Desai, S.K. Chavan. HISTO-MORPHOMETRIC STUDY
OF VARIOUS SEGMENTS OF VERTEBRAL ARTERY IN HUMAN
CADAVERS. Int J Anat Res 2019;7(1.1):6102-6107. DOI: 10.16965/
ijar.2018.415 